\title{
The value of information in asymmetric all-pay auctions
}

Citation for published version (APA):

Seel, C. (2014). The value of information in asymmetric all-pay auctions. Games and Economic Behavior, 86, 330-338. https://doi.org/10.1016/j.geb.2014.04.007

Document status and date:

Published: 01/07/2014

DOI:

10.1016/j.geb.2014.04.007

Document Version:

Publisher's PDF, also known as Version of record

Document license:

Taverne

Please check the document version of this publication:

- A submitted manuscript is the version of the article upon submission and before peer-review. There can be important differences between the submitted version and the official published version of record.

People interested in the research are advised to contact the author for the final version of the publication, or visit the DOI to the publisher's website.

- The final author version and the galley proof are versions of the publication after peer review.

- The final published version features the final layout of the paper including the volume, issue and page numbers.

Link to publication

\footnotetext{
General rights rights.

- You may freely distribute the URL identifying the publication in the public portal. please follow below link for the End User Agreement:

www.umlib.nl/taverne-license

Take down policy

If you believe that this document breaches copyright please contact us at:

repository@maastrichtuniversity.nl

providing details and we will investigate your claim.
}

Copyright and moral rights for the publications made accessible in the public portal are retained by the authors and/or other copyright owners and it is a condition of accessing publications that users recognise and abide by the legal requirements associated with these

- Users may download and print one copy of any publication from the public portal for the purpose of private study or research.

- You may not further distribute the material or use it for any profit-making activity or commercial gain

If the publication is distributed under the terms of Article $25 \mathrm{fa}$ of the Dutch Copyright Act, indicated by the "Taverne" license above, 
Note

\section{The value of information in asymmetric all-pay auctions}

\section{Christian Seel}

Maastricht University, School of Business and Economics, Department of Economics, P.O. Box 616, 6200 MD Maastricht, The Netherlands

\section{A R T I C L E I N F O}

Article history:

Received 14 October 2011

Available online 30 April 2014

\section{JEL classification:}

$\mathrm{C} 72$

$\mathrm{C} 73$

Keywords:

All-pay contests

Head start

Asymmetric information

\begin{abstract}
A B S T R A C T
This note analyzes a two-player all-pay auction with incomplete information. More precisely, one bidder is uncertain about the size of the initial advantage of his rival modeled as a head start in the auction.

I derive the unique Bayesian Nash equilibrium outcome for a large class of cumulative distribution functions of the head start. The stronger player generates an informational rent if and only if his head start distribution is not stochastically dominated by a uniform distribution. I identify why my results for an uncertain head start differ qualitatively from uncertainty about a cost function or a valuation.
\end{abstract}

(C) 2014 Elsevier Inc. All rights reserved.

\section{Introduction}

In many contests, one competitor has an initial advantage over her rivals. For instance, an incumbent firm possesses more knowledge about a new task when it is related to their previous work. In a contest for job promotion or an employee of the month award, a worker with a good reputation has an advantage over her competitors. Most representatives favor companies from their own district in public procurement.

In recent literature, these initial advantages are often modeled as deterministic head starts in all-pay contests (see, e.g., Konrad, 2002, 2004, Kaplan et al., 2003, Kirkegaard, 2012, and Siegel, 2014). In the above mentioned applications, however, a contestant is typically not perfectly informed about the size of the head start of a rival. For instance, a lobbyist might know that the politician who he tries to influence comes from the same district as another lobbyist. Yet, he can only guess by how much this lowers his prospects in the contest.

This note provides a two-player contest model which captures asymmetric information about a head start. More precisely, the size of the head start is drawn from a commonly known probability distribution, but only the player with the head start knows the realization. ${ }^{1}$ Each player submits a non-negative bid and pays costs equal to her bid. The player with the higher score realization, i.e., sum of the bid and the head start, receives a prize.

I fully characterize the unique Bayesian Nash equilibrium distributions over scores for generic strictly increasing and continuous distributions of the head start in Proposition 1. In general, the support of the scoring distribution of the uninformed player is a union of disjoint intervals. The "gaps" in her support are intervals on which a bid results in a negative expected profit for all bidding strategies of the informed player. Both players randomize their scores uniformly on the remaining intervals.

In the following, I analyze the value of private information about the head start. For this purpose, I compare the ex-ante (before the player with the head start learns the size) equilibrium payoff to the ex-ante equilibrium payoff if both players

E-mail address: c.seel@maastrichtuniversity.nl.

1 For a very interesting new approach to two-sided asymmetric information about abilities in all-pay contests, see Pérez-Castrillo and Wettstein (2013). 
learn the size of the head start (public information)-the difference is the ex-ante informational rent. Proposition 2 shows that the informed player generates no ex-ante informational rent if her head start distribution is strictly stochastically dominated by a uniform distribution. If the head start distribution is not weakly stochastically dominated by the same uniform distribution, the informed player generates an informational rent.

Based on this result, I analyze which "types" benefit most from having private information in the latter case. To do so, I derive the ex-interim informational rent, i.e., the value of private information after the informed player knows her type in Proposition 3. It turns out that a lower head start leads to a weakly higher ex-interim informational rent. Intuitively, a high probability of facing stronger types scares the uninformed player who therefore abstains from bidding with positive probability. Thus, the weaker types of the informed player get a higher payoff than in the public information case, since their probability of winning with a bid of zero increases.

In contrast to the result for an uncertain head start, Proposition 4 shows that for an uncertain valuation, the "stronger" player always gets an informational rent. Hence, the note derives a qualitative difference in the information value of a technological advantage (lower marginal cost or higher valuation) versus an incumbency advantage based on previous work or reputation (higher head start). For different realizations of the head start, all types of the stronger player value an increase in the winning probability equally, while the value of an increase in the winning probability differs across types for different valuations. This leads to a different equilibrium bidding behavior of the weaker player which, in turn, yields a qualitatively different result for the informational rents.

The informational rents quantify the gains of hiding or acquiring information in the current setting with one-sided private information. Moreover, in future work, the present game might be embedded as a subgame of a two-sided information acquisition game. The closed-form solutions for the informational rents are particularly useful for this approach, since they specify (continuation) payoffs for one subgame following an initial information acquisition stage uniquely.

\section{The model}

I consider a model with two risk-neutral players $i=1,2$ who have a common valuation $v$ for an object; $v$ is henceforth normalized to 1 . Player 1 enjoys a head start which is a realization of a random variable $\Theta$ with a commonly known, continuous cumulative distribution function (cdf) $H$. The cdf $H$ is strictly increasing on its support $[\underline{\theta}, \bar{\theta}]$, where $0 \leq \underline{\theta}<1$ and $H(\underline{\theta})=0$. Player 1 observes the realization, while player 2 only knows the distribution $H$ of the random variable $\Theta$, where $H(\theta)=\mathbb{P}(\Theta \leq \theta)$.

Both players simultaneously submit bids $b_{i} \in \mathbb{R}_{+}$and pay costs $c\left(b_{i}\right)=b_{i}$. A mixed strategy of player 1 assigns a probability distribution over bids to each possible draw from $H$. I denote a pure strategy of player 1 by $b_{1}(\theta)$. Given $b_{1}(\theta)$, player 2 perceives player 1's bid as a random variable $b_{1}(\Theta)$. A mixed strategy of player 2 is a probability distribution over bids.

The score realization of a player consists of the realization of the head start (which is always zero for player 2) plus the realization of the bid. The player with the higher score realization wins the object; ties are broken in favor of player $1 .{ }^{2}$ Given the strategy of player $i$, her score is a random variable $S_{i}$ with a distribution which I denote by $F_{i}$, where $F_{i}(s)=$ $\mathbb{P}\left(S_{i} \leq s\right)$. Taking the strategy of the other player as given, I denote the expected payoff of a bid by $\Pi_{1}\left(\theta, b_{1}\right)$ and $\Pi_{2}\left(b_{2}\right)$.

\section{The equilibrium}

As a benchmark, I briefly review the two-player equilibrium in the absence of uncertainty which is first established in Konrad (2002), Lemma 1. It contains the equilibrium without a head start as a special case.

Lemma 1. Assume player 1 has a deterministic head start of $\theta \in[0,1)$. In the unique Nash equilibrium distribution over scores, player 2 places an atom of size $\theta$ at 0 and player 1 places an atom of size $\theta$ at $\theta$. Both players randomize uniformly with density 1 on $(\theta, 1]$. The expected payoff of player 1 is $\theta$; the expected payoff of player 2 is 0 .

In the following, I discuss how results differ if the size of the head start is private information. To do so, I construct the generically unique Bayesian Nash equilibrium scoring distributions $F_{1}$ and $F_{2}$ of the game. Moreover, I derive a corresponding bidding strategy for player 1 ; player 2 randomizes his bid according to $F_{2}$.

Proposition 1. There exists a Bayesian Nash equilibrium such that $F_{1}(s)=\min \{H(s), s\}$ and $F_{2}(s)=\int_{0}^{1} \mathbf{1}_{\{H(y)<y\}} \mathrm{d} y+$ $\int_{0}^{s} \mathbf{1}_{\{H(y) \geq y\}} \mathrm{d} y$. An equilibrium strategy of player 1 is $b_{1}(\theta)=\max \{H(\theta)-\theta, 0\}$.

If $H(\theta)=\theta$ on a set of measure zero, the equilibrium cdf's $F_{1}$ and $F_{2}$ are unique.

Proof. The existence proof proceeds in two steps. I first show that $b_{1}(\theta)$ induces the scoring distribution $F_{1}$. In a second step, I show that no player wants to deviate from the derived strategy.

\footnotetext{
2 The set of equilibria does not depend on the tie-breaking rule.
} 

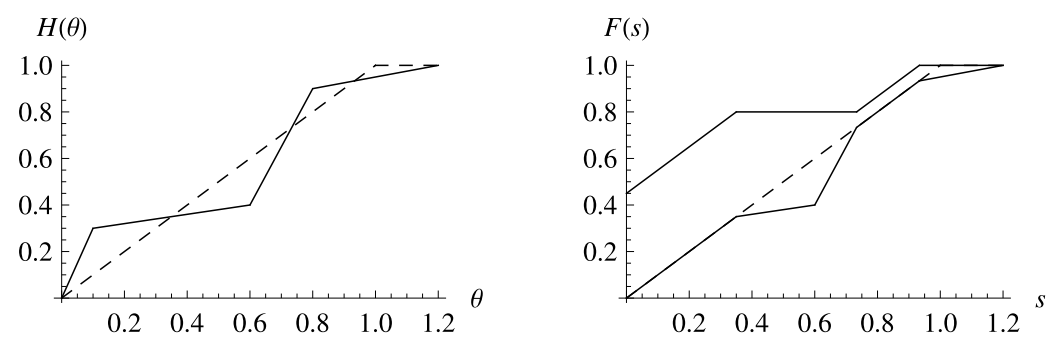

Fig. 1. The left panel shows an example of a head start distribution in the solid line and the uniform distribution on [0,1] in the dotted line. The right panel shows the unique equilibrium scoring distributions $F_{1}$ (lower solid line) and $F_{2}$ (upper solid line) for the head start distribution in the left panel.

Step 1: Since $H$ is continuous and strictly increasing on its support, it is also invertible on its support. Thus, I obtain

$$
\begin{aligned}
F_{1}(s) & =\mathbb{P}\left(S_{1} \leq s\right)=\mathbb{P}\left(\Theta+b_{1}(\Theta) \leq s\right)=\mathbb{P}(\max \{H(\Theta), \Theta\} \leq s) \\
& =\mathbb{P}\left(\{\Theta \leq s\} \cap\left\{\Theta \leq H^{-1}(s)\right\}\right)=\mathbb{P}\left(\Theta \leq \min \left\{s, H^{-1}(s)\right\}\right) \\
& = \begin{cases}H(s) & \text { if } s \leq H^{-1}(s) \\
H\left(H^{-1}(s)\right)=s & \text { if } s>H^{-1}(s)\end{cases} \\
& =\min \{H(s), s\} .
\end{aligned}
$$

Step 2: (i) For any two scores $s, s^{\prime}$ such that $s>s^{\prime}, F_{2}(s)-F_{2}\left(s^{\prime}\right) \leq s-s^{\prime}$. Hence,

$$
\Pi_{1}\left(\theta, b_{1}\right)=F_{2}\left(\theta+b_{1}\right)-b_{1} \leq F_{2}(\theta)=\Pi_{1}(\theta, 0),
$$

i.e., it is a best response to bid zero for each type $\theta$ of player 1 .

Assume $H(\theta)>\theta$ and $\theta^{\prime} \in[\theta, H(\theta)]$. Then $H\left(\theta^{\prime}\right) \geq H(\theta) \geq \theta^{\prime}$. As $H\left(\theta^{\prime}\right) \geq \theta^{\prime}$ for all $\theta^{\prime} \in[\theta, H(\theta)]$, I obtain $F_{2}(H(\theta))-$ $F_{2}(\theta)=H(\theta)-\theta$. Hence, $\Pi_{1}(\theta, H(\theta)-\theta)=\Pi_{1}(\theta, 0)$, i.e., it is a best response to bid $H(\theta)-\theta$ if $H(\theta)>\theta$.

(ii) By continuity of $F_{1}$, I obtain $\Pi_{2}\left(b_{2}\right)=F_{1}\left(b_{2}\right)-b_{2}$. Inserting $F_{1}$, I obtain $\Pi_{2}\left(b_{2}\right)=0$ whenever $F_{1}\left(b_{2}\right)=b_{2}$ and $\Pi_{2}\left(b_{2}\right)<0$ otherwise. Thus, any bid contained in the randomization of player 2 is a best response.

The uniqueness proof is relegated to Appendix A. It relies on a sequence of lemmas that are now commonplace in uniqueness proofs for static game theory with a continuous state space.

Before I proceed with the intuition for Proposition 1, I state two corollaries that are helpful in building this intuition. They refer to cases in which the head start distribution and the uniform distribution on $[0,1]$ can be ranked according to first-order stochastic dominance.

Corollary 1. Assume $H(\theta)>\theta$ for all $\theta \in(0,1)$. The unique Bayesian Nash equilibrium $c d f$ 's are $F_{i}(s)=\min \{s, 1\}$ for $i=1,2$.

Corollary 2. Assume $H(\theta)<\theta$ for all $\theta \in(0,1)$. The unique Bayesian Nash equilibrium cdf's are $F_{1}(s)=H(s)$ and $F_{2}(s)=1$ for all $s \geq 0$, i.e., both players always bid 0 .

In Corollary 1, the head start distribution is stochastically dominated by the uniform distribution, i.e., a high head start is relatively unlikely. In this case, the equilibrium scoring distributions are identical to the case without a head start (see Lemma 1 for $\theta=0$ ). Intuitively, player 2 does not see player 1's head start as a sufficient thread to change his strategy.

On the other hand, Corollary 2 considers cases in which high head start values are very likely. For player 2, it is not even profitable to choose a positive bid if every type of player 1 bids 0 , since his expected disadvantage through the head start is too high. Hence, he chooses the most cautious strategy, i.e., a bid of zero. Player 1 also bids zero and wins the contest with probability one.

The more general statement in Proposition 1 also includes cases in which the head start distribution and the uniform distribution on $[0,1]$ cannot be ranked in terms of first-order stochastic dominance. For an illustration of the equilibrium in such a case, see Fig. 1.

The uninformed player randomizes on all intervals for which he makes a positive profit if the informed player bids zero independently of her type. On the remaining intervals, the uninformed player does not bid with positive probability, because any bid would lead to an expected loss for all strategies of the informed player.

Some remarks about the uniform distribution on $[0,1]$ are in order, since it appears in the genericity condition for uniqueness in Proposition 1 and it separates Corollary 1 from Corollary 2. In particular, the bidding behavior of player 2 exhibits a bid bifurcation at $H(\theta)=\theta$, i.e., a qualitative change in behavior. ${ }^{3}$ As an illustration, consider the family of distri-

\footnotetext{
${ }^{3}$ For other results about bid bifurcation in all-pay auctions, see Noussair and Silver (2006) and Parreiras and Rubinchik (2010).
} 
bution functions $H_{\epsilon}(\theta)=(1+\epsilon) \theta$. For any $\epsilon<0$, player 2 bids zero with probability 1 . For any $\epsilon>0$, however, player 2 randomizes his bid uniformly on $[0,1]$. Therefore, the payoff of player 1 is not continuous in her head start distribution.

If the head start distribution is the uniform distribution on $[0,1]$, the equilibrium is no longer unique. For instance, in one equilibrium each type of player 1 bids zero and player 2 randomizes uniformly on [0,1]. In a second equilibrium, each type of player 1 bids zero and player 2 bids zero with probability 1 . This multiplicity occurs because the equilibrium scoring distribution of player 1 does not contain positive bids. Otherwise, the second equilibrium would be ruled out, because each type of player 1 would prefer to lower her bid, since she would still win with probability one at a lower cost. A similar intuition holds whenever $H(\theta)=\theta$ on a set of positive measure.

In the next step, I compare the ex-ante difference in expected payoffs for the equilibria I have obtained under private information to the benchmark of public information. Throughout this analysis, I assume that under public information both players observe the realization of player 1's head start. I refer to the ex-ante difference in expected equilibrium payoffs as the ex-ante informational rent.

Proposition 2. Player 1 generates a strictly positive ex-ante informational rent if $H(\theta)<\theta$ for some $\theta \in(0,1)$. However, if $H(\theta)>\theta$ for all $\theta \in(0,1)$, player 1 does not obtain an ex-ante informational rent. The ex-ante informational rent of player 2 is always 0 .

Thus, private information about the head start is not always beneficial. This finding is in contrast to the existing literature on all-pay auctions with incomplete information in which the stronger player always benefits from having private information. I give a more detailed explanation for this new feature after the analysis of the corresponding model with uncertainty about one valuation.

In the last part of this section, I analyze the gain from having private information ex-interim, i.e., after player 1 learns her type compared to the public information case.

Proposition 3. Assume $H(\theta)=\theta$ on a set of measure zero. Type $\theta$ of player 1 generates an ex-interim informational rent of $\max \left\{\int_{\theta}^{1} \mathbf{1}_{\{H(y)<y\}} \mathrm{d} y, 0\right\}$.

Note that $\int_{\theta}^{1} \mathbf{1}_{\{H(y)<y\}} \mathrm{d} y$ is weakly decreasing in $\theta$. Thus, the ex-interim informational rent is the maximum of a weakly decreasing function and a constant and thereby also weakly decreasing. Intuitively, the risk of facing high types leads player 2 to bid more cautiously than under public information. In turn, this increases the payoff of the weaker types of player 1.

Remark 1. Throughout this section, I have normalized the common valuation $v$ to 1 for notational convenience. If one inverts this normalization, the benchmark for comparison is the uniform distribution on $[0, v]$ with density $\frac{1}{v}$ instead of the uniform distribution on $[0,1]$ with density 1 . This does not change the results qualitatively.

\section{Uncertainty about one valuation}

In this section, I contrast the main results of this note with the corresponding results for an all-pay auction in which player 1 has a higher valuation than player $2 .{ }^{4}$ More precisely, player 2 has a valuation of $v_{2}$. The valuation of player 1 is the realization of a random variable $V$ with a commonly known, continuous distribution $J$ which is strictly increasing on its support $\left[v_{2}, \bar{v}_{1}\right]$. As before, players compete in an all-pay auction with costs $c\left(b_{i}\right)=b_{i}{ }^{5}$ No player has a head start. I denote a pure strategy of player 1 by $b_{1}\left(v_{1}\right)$ and the score it induces by $b_{1}(V)$. I denote the scoring distributions by $G_{i}: \mathbb{R}_{+} \rightarrow[0,1]$ and associated density functions by $g_{i}$.

For the benchmark case in which both players know the realization $v_{1}$, Hillman and Riley (1989) obtain the following result:

Lemma 2. (See Hillman and Riley, 1989.) In the unique Nash equilibrium, $G_{1}(s)=\min \left\{\frac{s}{v_{2}}, 1\right\}$ and $G_{2}(s)=\min \left\{\frac{v_{1}-v_{2}}{v_{1}}+\frac{s}{v_{1}}, 1\right\}$. Player 1 obtains a profit of $v_{1}-v_{2}$ and player 2 obtains a profit of 0.

In the following, I consider the private information case. ${ }^{6}$

Proposition 4. In any Bayesian Nash equilibrium, $G_{1}(s)=\min \left\{\frac{s}{v_{2}}, 1\right\}$ and

\footnotetext{
4 See, e.g., Amann and Leininger (1996), Moldovanu and Sela (2001), and Kirkegaard (2012) for results on two-sided incomplete information.

5 To determine their best responses, players only care about the relationship between valuation-cost ratio and winning probability. Hence, a higher valuation of a player is strategically equivalent to a lower (marginal) cost.

6 In a recent contribution, Morath and Münster (2013) prove a similar result for the case in which $H$ is continuously differentiable and strictly increasing on $\left[0, \bar{v}_{1}\right]$.
} 


$$
G_{2}(s)= \begin{cases}1-\int_{s}^{v_{2}} \frac{1}{J^{-1}\left(\frac{y}{v_{2}}\right)} \mathrm{d} y & \text { if } s \in\left[0, v_{2}\right], \\ 1 & \text { if } s>v_{2} .\end{cases}
$$

The unique equilibrium strategy of player 1 is $b_{1}\left(v_{1}\right)=v_{2} J\left(v_{1}\right)$. Player 1 obtains an ex-ante informational rent and an ex-interim informational rent for each type $v_{1}<\bar{v}_{1}$. The informational rent of player 2 is zero.

Intuitively, player 2 uses a randomization which makes it optimal for each type $v_{1}$ of player 1 to bid $v_{2} J\left(v_{1}\right)$. Every type $v_{1}<\bar{v}_{1}$ makes a strictly higher ex-interim profit than in the benchmark case of public information. Thus, differing from the analysis for an uncertain head start, player 1 can always use her private information to her advantage.

\section{Discussion of the main results}

The main results illustrate the structural differences between incomplete information about previous work effort which can be used for a new task or a higher reputation (head start) and a better technology or higher motivation to win (lower costs or higher valuation). For the latter case, the value of an increase in the winning probability differs for each type of the informed player. In equilibrium, the uninformed player randomizes with a decreasing density on a connected support. Thereby, he guarantees a unique bidding strategy of his rival whose bids are increasing in her valuation. For an uncertain head start, however, all types of the informed player value an increase in the winning probability equally. Thus, the uninformed player randomizes with the same density whenever he chooses to randomize with positive density.

However, the uninformed player only randomizes on intervals for which he makes a positive profit for a zero bid of the informed player, i.e., if $H(\theta)>\theta$. Hence, there is a qualitative change in the bidding behavior of the uninformed player depending on whether the head start distribution lies (slightly) above or (slightly) below the uniform, i.e., bid bifurcation occurs at the uniform distribution.

From an empirical point of view, an outside observer would not be able to tell whether a player had a head start if the head start distribution is dominated by the uniform distribution on $[0,1]$. On the other hand, a non-zero bid of both players entails that the head start distribution is not stochastically dominated by the uniform distribution. Hence, observing the equilibrium outcome allows an outside observer to (partially) learn about the information structure.

For the case of one-sided asymmetric information, the informational rent results directly answer a question regarding the value of hiding or acquiring information. In a lobbying contest, for instance, an out-of-state company would like to know how beneficial it is to learn her opponent's advantage; the home company would want to know how much hiding this information is worth. In (public) procurement, firms are also eager to understand possible benefits from learning or hiding the size of an incumbency advantage.

Moreover, the present game is a first step towards solving multistage games with an additional initial two-sided information acquisition or information sharing decision. The closed-form solutions in this note determine the payoffs in the subgame after a one-sided information acquisition or sharing decision uniquely. ${ }^{7}$

\section{A potentially negative head start}

This section considers head start distributions whose support contains negative values. Intuitively, the player who possesses private information might have an initial disadvantage. For instance, one might think of a procurement contest in which an incumbent builds upon her innovation from a previous contest. In this case, a new competitor knows the quality of the incumbent's innovation, but the incumbent is not perfectly informed how difficult it is for the new competitor to replicate the innovation.

As before, let $H$ denote the head start distribution. I assume that $H$ is strictly increasing on its support [ $\theta \underline{\theta}, \bar{\theta}]$, where $\underline{\theta}<0$. Note that $\bar{\theta}$ can be positive. Moreover, the cdf $H$ is differentiable with a density $h$ for which $h(\theta)=1$ on a set of measure zero. This condition is similar, yet slightly stronger than the genericity condition for the uniqueness part in Proposition 1. Moreover, I now impose it before the main result, since both the existence and the uniqueness proof rely on this condition.

If $\bar{\theta} \leq-1$, there exists a unique equilibrium in which both players bid zero for any type. Thus, I henceforth restrict attention to the more interesting case $\bar{\theta}>-1$.

Define $\theta^{*}$ as the solution to $-\theta+\int_{0}^{1} \mathbf{1}_{\{H(\theta)+y<H(y)\}} \mathrm{d} y=1$. I show in Lemma 6 in Appendix A that $\theta^{*}$ is unique and that it lies in the interval $[-1,0]$. The point $\theta^{*}$ is crucial for the following proposition:

Proposition 5. A Bayesian Nash equilibrium exists. In any equilibrium,

$$
F_{1}(s)= \begin{cases}H(s) & \text { if } s \leq \theta^{*}, \\ H\left(\theta^{*}\right) & \text { if } \theta^{*}<s \leq 0 \\ \min \left\{H\left(\theta^{*}\right)+s, H(s)\right\} & \text { if } s>0\end{cases}
$$

\footnotetext{
7 For the case of uncertain valuations, Morath and Münster (2013) and Kovenock et al. (2014) model the value of information acquisition and information sharing more explicitly.
} 
and

$$
F_{2}(s)= \begin{cases}0 & \text { if } s<0, \\ -\theta^{*}+\int_{0}^{s} \mathbf{1}_{\left\{H\left(\theta^{*}\right)+y<H(y)\right\}} \mathrm{d} y & \text { if } s \geq 0 .\end{cases}
$$

An equilibrium strategy of player 1 is

$$
b_{1}(\theta)= \begin{cases}\max \left\{H(\theta)-H\left(\theta^{*}\right)-\theta, 0\right\} & \text { if } \theta>\theta^{*}, \\ 0 & \text { if } \theta \leq \theta^{*} .\end{cases}
$$

The shape of the equilibrium distributions in Proposition 5 is similar to those in Proposition 1 . The additional constant $H\left(\theta^{*}\right)$ in the cdf $F_{1}$ illustrates that the lowest types of player 1 might abstain from bidding. The profit of player 2 is now equal to the mass of types of player 1 who choose a negative score realization. Since any bid of player 2 has to ensure the same payoff as bidding zero, he only randomizes with positive probability if $H\left(\theta^{*}\right)+\theta<H(\theta)$ instead of $\theta<H(\theta)$ which ensures a payoff of zero in Proposition 1.

In equilibrium, the types $\theta \in\left(\theta^{*}, 0\right)$ of player 1 make strictly positive expected profits, while they would make zero profits under public information. Similarly, any type $\theta \in[0,1]$ could ensure the public information payoff $\theta$ with a bid $1-\theta$, since $\Pi_{1}(\theta, 1-\theta)=F_{2}(1)-(1-\theta)=1-(1-\theta)=\theta$. Hence, player 1 still generates an ex-ante informational rent from her private information and no type of player 1 is worse off by possessing private information.

\section{Acknowledgments}

I am indebted to the anonymous reviewers for a myriad of comments and suggestions which have greatly improved the quality of this note. Moreover, I would like to thank Sebastian Ebert, Susanne Goldlücke, Dan Kovenock, Thomas Kruse, Matthias Lang, Wolfgang Leininger, Benny Moldovanu, Johannes Münster, Volker Nocke, David Pérez-Castrillo, Sven Rady, Ron Siegel, Philipp Strack, Nora Szech, Tymon Tatur, Ernst-Ludwig von Thadden, Cédric Wasser, and seminar participants in Aachen, Barcelona, Berlin, Bielefeld, Cologne, Düsseldorf, and Vallendar for inspiring discussions and comments.

\section{Appendix A}

In order to complete the proof of Proposition 1, I derive a sequence of auxiliary results that have to hold in any (Bayesian Nash) equilibrium. The proof of the next lemma is standard in this literature and thus omitted. ${ }^{8}$

Lemma 3. In any equilibrium, $F_{2}$ is continuous on $\mathbb{R}_{+}$, i.e., player 2 does not place an atom at any $s>0$.

Lemma 4. If player 2 randomizes with a positive density $f_{2}$ over an interval $I=[a, b]$, then $f_{2}(s) \leq 1$ almost everywhere on $I$.

Proof. Assume player 2 randomizes with positive density on $I$. By optimality for player 2 , every bid $b_{2} \in I$ leads to the same payoff which implies $f_{1}(s)=1$ almost everywhere on $I$. Hence, $f_{2}(s) \leq 1$ almost everywhere on $I$, since player 1 would strictly prefer to increase his score realization on an interval $(c, d) \subset I$ if $f_{2}(s)>1$ for all $s \in(c, d)$; this contradicts optimality of player 1 's randomization almost everywhere on $I$.

Lemma 5. Player 2 obtains a payoff of zero.

Proof. As any bid above 1 leads to a negative payoff for player $2, F_{2}(1)=1$ in any equilibrium. Moreover, player 2 places no mass points above 0 by Lemma 3 and $f_{2}(s) \leq 1$ almost everywhere by Lemma 4 . As $F_{2}(1)=F_{2}(0)+\int_{0}^{1} f_{2}(y) d y=1$, player 2 either places a mass point at zero or he randomizes uniformly with $f_{2}(s)=1$ almost everywhere on the interval $[0,1]$. Since all equilibrium bids yield the same payoff for player 2 , he makes a payoff of $\Pi_{2}(0)=0$.

Proof of Proposition 1. The existence part has already been shown in the main text. In the following, I prove the uniqueness part:

Case 1: Assume $H(\theta)<\theta$. Then, by continuity of $H$, there exists an $\epsilon>0$ such that player 2 does not bid $b_{2} \in[\theta, \theta+\epsilon]$, because

$$
\Pi_{2}(\theta) \leq F_{1}(\theta)-\theta \leq H(\theta)-\theta<0 .
$$

8 All omitted proofs and steps are available from the author upon request. 
Since $f_{2}(s) \leq 1$ for almost all $s \geq 0$ by Lemma 4 and $f_{2}(s)=0$ for $s \in[\theta, \theta+\epsilon]$, I obtain

$$
\Pi_{1}(\theta, 0)=F_{2}(\theta)>F_{2}\left(b_{1}+\theta\right)-b_{1}=\Pi_{1}\left(\theta, b_{1}\right)
$$

for all $b_{1}>0$. Hence, player 1 bids 0 if $H(\theta)<\theta$.

Case 2: Assume $H(\theta)>\theta$. By the zero-profit condition for player 2 (Lemma 5 ), $F_{1}(s) \leq s$ for all $s \geq 0$. By contradiction, assume there exists an $\epsilon>0$ such that $F_{1}(s)<s$ for all $s \in[\theta, \theta+\epsilon)$. Then $F_{2}(s)$ is constant for $s \in[\theta, \theta+\epsilon)$. Recall that $f_{2}(s) \leq 1$ for almost all $s>0$ by Lemma 4. Hence, if player 1 has a head start $\theta^{\prime} \leq \theta$, she does not choose a score above $\theta$, because

$$
\Pi_{1}\left(\theta^{\prime}, \theta-\theta^{\prime}\right)=F_{2}(\theta)-\left(\theta-\theta^{\prime}\right)>F_{2}(\theta+\epsilon)-\left(\theta+\epsilon-\theta^{\prime}\right)=\Pi_{1}\left(\theta^{\prime}, \theta-\theta^{\prime}+\epsilon\right) .
$$

for all $\epsilon>0$. Hence, $F_{1}(\theta)=H(\theta)>\theta$ which contradicts $F_{1}(s)<s$ for all $s \in[\theta, \theta+\epsilon)$.

Since $H(\theta)=\theta$ on a set of measure zero, the bidding strategy in this case has no impact on the scoring distribution Hence, the scoring distribution of player 1 is unique.

As shown in Case 2, for any $\theta^{\prime}$ such that $H\left(\theta^{\prime}\right)>\theta^{\prime}, F_{1}\left(\theta^{\prime}\right)=\theta^{\prime}$. Thus,

$$
\theta^{\prime}=F_{1}\left(\theta^{\prime}\right)=\mathbb{P}\left(\Theta+b_{1}(\Theta) \leq \theta^{\prime}\right)<\mathbb{P}\left(\Theta \leq \theta^{\prime}\right)=H\left(\theta^{\prime}\right) .
$$

Hence, there exists a type $\theta^{\prime \prime}<\theta^{\prime}$ of player 1 who bids $b_{1}$ such that $\theta^{\prime \prime}+b_{1}>\theta^{\prime}$. To ensure optimality of the bid $b_{1}$, $f_{2}(\theta)=1$ for almost all $\theta \in\left[\theta^{\prime \prime}, b_{1}+\theta^{\prime \prime}\right]$. Thus, for any $\theta^{\prime}$ such that $H\left(\theta^{\prime}\right)>\theta^{\prime}, f_{2}(\theta)=1$ for almost all $\theta$ in some $\epsilon$-neighborhood around $\theta^{\prime}$. Together with $f_{2}(\theta)=0$ whenever $H(\theta)<\theta$ (see Case 1 ), this uniquely determines the distribution of player 2 .

Proof of Proposition 2. If $H(\theta)>\theta$ for all $\theta \in(0,1)$, player 2 randomizes uniformly on $(0,1)$ by Corollary 1 . Thus, the expected payoff for player 1 is equal to the size of her head start $\theta$ for all $\theta$, which equals the payoff in Lemma 1 .

Note that I can use the results of Lemmas 3-5 and Case 1 in the uniqueness proof of Proposition 1 for this proof, since the proofs do not use the condition $H(\theta)=\theta$ on a set of measure zero. Player 2 places no atom at 1 by Lemma 3 and never bids above 1 , since this would lead to a negative payoff. Hence, for all $\theta$, player 1 can ensure her public information payoff of $\theta$ by bidding $1-\theta$. If $H(\theta)<\theta$ on some interval $(a, b)$, player 2 does not bid on the interval ( $a, b)$, because $\Pi_{2}(\theta) \leq F_{1}(\theta)-\theta \leq H(\theta)-\theta<0 \leq \Pi_{2}(0)$ for all $\theta \in(a, b)$. In combination with Lemma 4 and $F_{2}(1)=1$, I obtain $F_{2}(s)>s$ for all $s \in(a, b)$. Hence, for any $\theta \in(a, b)$, a bid of 0 yields player 1 a payoff which is strictly higher than her payoff under public information. Hence, player 1 obtains an ex-ante informational rent.

The result for player 2 follows immediately from Lemma 1 and Lemma 5.

Proof of Proposition 3. Since $F_{2}$ is continuous and $f_{2}(s) \leq 1$ for almost all $s$, a bid of zero is optimal for any type $\theta$ of player 1. Hence, it suffices to compare the payoff of type $\theta$ from bidding 0 to the payoff of type $\theta$ under complete information (see Lemma 1 ). For any type $\theta \in[0,1]$, I obtain

$$
\begin{aligned}
\Pi_{1}(\theta, 0)-\theta & =F_{2}(\theta)-\theta=\int_{0}^{1} \mathbf{1}_{\{H(y)<y\}} \mathrm{d} y+\int_{0}^{\theta} \mathbf{1}_{\{H(y) \geq y\}} \mathrm{d} y-\theta \\
& =\int_{0}^{1} \mathbf{1}_{\{H(y)<y\}} \mathrm{d} y-\int_{0}^{\theta} \mathbf{1}_{\{H(y)<y\}} \mathrm{d} y=\int_{\theta}^{1} \mathbf{1}_{\{H(y)<y\}} \mathrm{d} y .
\end{aligned}
$$

The formula in Proposition 3 follows, since the payoff of types $\theta>1$ is 1 in any equilibrium.

Proof of Proposition 4. Since $J$ is invertible on its support, for all $s \in\left[0, v_{2}\right]$, the bidding strategy $b_{1}\left(v_{1}\right)=v_{2} J\left(v_{1}\right)$ leads to the score distribution

$$
G_{1}(s)=\mathbb{P}\left(b_{1}(V) \leq s\right)=\mathbb{P}\left(V \leq b_{1}^{-1}(s)\right)=J\left(b_{1}^{-1}(s)\right)=J\left(J^{-1}\left(\frac{s}{v_{2}}\right)\right)=\frac{s}{v_{2}} .
$$

The next step establishes uniqueness of player 1's optimal bidding strategy. Player 2 never bids above $v_{2}$ in equilibrium, since this would entail a negative payoff. Thus, player 1 can guarantee herself a payoff of $v_{1}-v_{2}$ by bidding $v_{2}$. A similar argument to that in the proof of Lemma 3 shows that $G_{1}$ and $G_{2}$ are continuous with $G_{1}(0)=0$. A similar argument to that in the proof Lemma 4 with reversed player roles entails $g_{1}(s) \leq \frac{1}{v_{2}}$ almost everywhere. As player 1 places no mass point and $G_{1}\left(v_{2}\right)=1$, this implies $G_{1}\left(v_{2}\right)=\int_{0}^{v_{2}} g_{1}(s) \mathrm{d} s=1$. Thus, $G_{1}(s)=\min \left\{\frac{s}{v_{2}}, 1\right\}$. To obtain uniqueness of the strategy of player 1, it remains to show that her bid is increasing in her valuation in any equilibrium. This, however, is straightforward, since a higher bid entails the same additional cost for each type, but increases the winning probability and thereby the gain of a type with a higher valuation. 
The next step establishes uniqueness of player 2's equilibrium distribution. The optimization problem of player 1 entails

$$
v_{1} G_{2}\left(b_{1}\left(v_{1}\right)\right)-b_{1}\left(v_{1}\right) \geq v_{1} G_{2}\left(\hat{b}_{1}\right)-\hat{b}_{1}
$$

for all $v_{1}, \hat{b}_{1}$. For the rest of the proof, I denote $b_{1}=b_{1}\left(v_{1}\right)$. From Eq. (1), I obtain two conditions:

$$
\frac{G_{2}\left(b_{1}\right)-G_{2}\left(\hat{b}_{1}\right)}{b_{1}-\hat{b}_{1}} \geq \frac{1}{v_{1}} \quad \forall \hat{b}_{1}<b_{1}
$$

and

$$
\frac{G_{2}\left(b_{1}\right)-G_{2}\left(\hat{b}_{1}\right)}{b_{1}-\hat{b}_{1}} \leq \frac{1}{v_{1}} \quad \forall \hat{b}_{1}>b_{1} .
$$

At the same time,

$$
\hat{v}_{1} G_{2}\left(\hat{b}_{1}\right)-\hat{b}_{1} \geq \hat{v}_{1} G_{2}\left(b_{1}\right)-b_{1},
$$

where $\hat{b}_{1}=b_{1}\left(\hat{v}_{1}\right)$. Thus, Eq. (4) implies

$$
\frac{1}{\hat{v}_{1}} \geq \frac{G_{2}\left(b_{1}\right)-G_{2}\left(\hat{b}_{1}\right)}{b_{1}-\hat{b}_{1}} \quad \forall b_{1}>\hat{b}_{1}
$$

and

$$
\frac{1}{\hat{v}_{1}} \leq \frac{G_{2}\left(b_{1}\right)-G_{2}\left(\hat{b}_{1}\right)}{b_{1}-\hat{b}_{1}} \quad \forall b_{1}<\hat{b}_{1} .
$$

By continuity of $G_{2}$, I obtain

$$
\lim _{\hat{b}_{1} \uparrow b_{1}} \frac{G_{2}\left(b_{1}\right)-G_{2}\left(\hat{b}_{1}\right)}{b_{1}-\hat{b}_{1}}=\frac{1}{v_{1}}=\lim _{\hat{b}_{1} \downarrow b_{1}} \frac{G_{2}\left(b_{1}\right)-G_{2}\left(\hat{b}_{1}\right)}{b_{1}-\hat{b}_{1}} .
$$

The first equality follows from (2) and (5), while the second one follows from ( 3 ) and (6). Hence, $G_{2}$ is differentiable on $\left(0, v_{2}\right)$ with the derivative $g_{2}\left(b_{1}\left(v_{1}\right)\right)=\frac{1}{v_{1}}$. Thus, on $\left(0, v_{2}\right)$, I obtain $g_{2}(s)=\frac{1}{b_{1}^{-1}(s)}=\frac{1}{J^{-1}\left(\frac{s}{v_{2}}\right)}$. Imposing the boundary condition $G_{2}\left(v_{2}\right)=1$, the differential equation yields

$$
G_{2}(s)=1-\int_{0}^{v_{2}} \frac{1}{J^{-1}\left(\frac{y}{v_{2}}\right)} \mathrm{d} y+\int_{0}^{s} \frac{1}{J^{-1}\left(\frac{y}{v_{2}}\right)} \mathrm{d} y=1-\int_{s}^{v_{2}} \frac{1}{J^{-1}\left(\frac{y}{v_{2}}\right)} \mathrm{d} y
$$

as the unique solution. The second-order condition shows that the bid $b_{1}\left(v_{1}\right)=v_{2} J\left(v_{1}\right)$ is indeed the maximizer for all $v_{1} \in\left[v_{2}, \bar{v}_{1}\right]$.

Player 1 obtains an informational rent in equilibrium, since bidding $b_{1}\left(v_{1}\right)=v_{2}$ would yield him the public information payoff $v_{1}-v_{2}$, but any equilibrium bid yields a strictly higher payoff for all types $v_{1} \in\left[v_{2}, \bar{v}_{1}\right.$ ). Player 2 obtains a payoff of 0 under both informational assumptions.

Lemma 6. There exists a unique point $\theta^{*} \in[-1,0]$ such that

$$
-\theta+\int_{0}^{1} \mathbf{1}_{\{H(\theta)+y<H(y)\}} \mathrm{d} y=1 .
$$

Proof. For $\theta=-1$,

$$
-\theta+\int_{0}^{1} \mathbf{1}_{\{H(\theta)+y<H(y)\}} \mathrm{d} y=1+\int_{0}^{1} \mathbf{1}_{\{H(-1)+y<H(y)\}} \mathrm{d} y \geq 1 .
$$

On the other hand, for $\theta=0$,

$$
-\theta+\int_{0}^{1} \mathbf{1}_{\{H(\theta)+y<H(y)\}} \mathrm{d} y=\int_{0}^{1} \mathbf{1}_{\{H(0)+y<H(y)\}} \mathrm{d} y \leq 1 .
$$


Moreover, $-\theta$ is continuous and strictly monotone decreasing and $\int_{0}^{1} \mathbf{1}_{\{H(\theta)+y<H(y)\}} \mathrm{d} y$ is weakly monotone decreasing in $\theta$. The assumption $h(y)=1$ on a set of measure zero implies $H(y)-y=H(\theta)$ on a set of measure zero for all $\theta$. Hence, $\int_{0}^{1} \mathbf{1}_{\{H(\theta)+y<H(y)\}} \mathrm{d} y$ is also continuous. Thus, $-\theta+\int_{0}^{1} \mathbf{1}_{\{H(\theta)+y<H(y)\}} \mathrm{d} y$ is continuous and strictly monotone decreasing. By the Intermediate Value Theorem, there exists a unique point $\theta^{*} \in[-1,0]$ for which $-\theta+\int_{0}^{1} \mathbf{1}_{\{H(\theta)+y<H(y)\}} \mathrm{d} y=1$.

Proof of Proposition 5. The existence proof proceeds in three steps. Steps 1 and 2 are almost identical to Steps 1 and 2 of Proposition 1 with $H(\theta)-H\left(\theta^{*}\right)$ instead of $H(\theta)$ and thus omitted.

Step 3: The function $F_{2}$ has to be a cdf. Since player 2 never bids above one in equilibrium, I obtain $F_{2}(1)=\theta^{*}+$ $\int_{0}^{1} \mathbf{1}_{\left\{H\left(\theta^{*}\right)+y<H(y)\right\}} \mathrm{d} y=1$. Thus, for $\theta^{*}$ as defined in the main text, the function $F_{2}$ is a cdf.

The uniqueness proof is similar to the uniqueness proof of Proposition 1 and thus omitted.

\section{References}

Amann, E., Leininger, W., 1996. Asymmetric all-pay auctions with incomplete information: the two-player case. Games Econ. Behav. 14, 1-18. Hillman, A.L., Riley, J.G., 1989. Politically contestable rents and transfers. Econ. Polit. 1, 17-39.

Kaplan, T.R., Luski, I., Wettstein, D., 2003. Innovative activity and sunk cost. Int. J. Ind. Organ. 21, 1111-1133.

Kirkegaard, R., 2012. Favoritism in asymmetric contests: head starts and handicaps. Games Econ. Behav. 76, $226-248$.

Konrad, K., 2002. Investment in the absence of property rights: the role of incumbency advantages. Europ. Econ. Rev. 46, $1521-1537$.

Konrad, K., 2004. Inverse campaigning. Econ. J. 114, 69-82.

Kovenock, D., Morath, F., Münster, J., 2014. Information sharing in contests. J. Econ. Manag. Strategy, forthcoming.

Moldovanu, B., Sela, A., 2001. The optimal allocation of prizes in contests. Amer. Econ. Rev. 91, 542-558.

Morath, F., Münster, J., 2013. Information acquisition in conflicts. Econ. Theory 54, 99-129.

Noussair, C., Silver, J., 2006. Behavior in all-pay auctions with incomplete information. Games Econ. Behav. 55, 189-206.

Parreiras, S.O., Rubinchik, A., 2010. Contests with three or more heterogeneous agents. Games Econ. Behav. 68, 703-715.

Pérez-Castrillo, D., Wettstein, D., 2013. Innovation contests. Mimeo.

Siegel, R., 2014. Asymmetric contests with head starts and non-monotonic costs. Amer. Econ. J. Macroecon., forthcoming. 